\title{
Integrins Are the Necessary Links to Hypertrophic Growth in Cardiomyocytes
}

\author{
Rebecca K. Harston ${ }^{1,2}$ and Dhandapani Kuppuswamy ${ }^{1}$ \\ ${ }^{1}$ Cardiology Division, Department of Medicine, Gazes Cardiac Research Institute, Medical University of South Carolina, Charleston, \\ SC 29425-2221, USA \\ ${ }^{2}$ Advanced BioScience Laboratories, Inc., 5510 Nicholson Lane, Kensington, MD 20895, USA
}

Correspondence should be addressed to Dhandapani Kuppuswamy, kuppusd@musc.edu

Received 21 October 2010; Accepted 15 December 2010

Academic Editor: Wan-Wan Lin

Copyright ( 2011 R. K. Harston and D. Kuppuswamy. This is an open access article distributed under the Creative Commons Attribution License, which permits unrestricted use, distribution, and reproduction in any medium, provided the original work is properly cited.

\begin{abstract}
To compensate for hemodynamic overload of the heart, an event which stretches the myocardium, growth and survival signaling are activated in cardiac muscle cells (cardiomyocytes). Integrins serve as the signaling receptors of cardiomyocytes responsible for mechanotransduction toward intracellular signaling. The main integrin heterodimers on the cardiomyocyte surface are $\alpha_{5} \beta_{1}$ and $\alpha_{v} \beta_{3}$, and elimination of either $\beta_{1}$ or $\beta_{3}$ integrins impedes pressure-induced hypertrophic signaling and leads to increased mortality. The growth signaling pathways downstream of $\beta_{1}$ and $\beta_{3}$ integrins are well characterized. However, new integrin pathways responsible for inhibiting apoptosis induced by hemodynamic overload are emerging. $\beta_{1}$ and $\beta_{3}$ integrins activate differential survival signaling, yet both integrins initiate survival signaling downstream of ubiquitination and the kinase pathway including phosphoinositol-3-kinase (PI3K)/Akt. Further characterization of these integrin-signaling mechanisms may lead to drug targets to prevent decompensation to heart failure.
\end{abstract}

\section{Cardiac Hypertrophy}

Cardiac hypertrophy begins as a compensatory response to hemodynamic overload, such as from increased pressure with hypertension. Several cell types are present in the ventricular wall that provide diverse contributions for optimal organ performance. Cardiomyocytes are the muscle cells in the heart which make up myofibrils that contract to pump blood to the body. Like skeletal muscle cells, cardiomyocytes are striated, but only cardiomyocytes have intercalated discs, which connect adjacent cells and help them simultaneously contract. While all cell types in the heart differentially respond to hemodynamic changes, the cardiomyocytes ultimately regulate cardiac performance through pump function and must change intracellular signaling pathways most substantially.

Hypertrophy is defined by the increase in protein synthesis, cardiomyocyte size, and cytoskeletal remodeling, which are important to increase the ventricle wall thickness to normalize against the mechanical stress [1] and optimize contractility (reviewed in [2]). Cardiomyocytes are terminally differentiated and, therefore, have limited proliferative potential. Thus, cell growth and initiation of survival signaling are their means of increasing ventricle muscle mass. As cardiomyocytes reenter a growth phase not initiated since development, there is an immediate early gene induction of proto-oncogenes, and heat shock protein genes are activated first, followed by induction of a "fetal" gene program similar to developmental expression patterns. However, hypertrophic growth often results in heart failure by a poorly understood mechanism when the heart can no longer compensate for sustained pressure overload (PO). Hypertension is the leading cause for heart disease and stroke in the United States and thus remains an important area to study for a beneficial impact on human health.

\section{Hypertrophic Growth by Integrin Activation}

Hemodynamic overload of the heart instigates many stimuli received by cardiomyocytes for induction of growth, 
including neurohormonal changes, mobilization of growth factors, stretch of the myocardium, and extracellular matrix (ECM) remodeling. Many of these upstream events either utilize or synergize integrin receptors to initiate growth and survival signaling. Cardiomyocytes require tight regulatory processes to elicit signaling changes in response to hypertrophic stimuli. In addition, cytoskeletal dynamics must be able to change, but remain functional in order for each cardiomyocyte to enlarge, add sarcomeres, and extend their cell borders within the heart tissue [1]. Targeted protein synthesis, which is required for growth, is increased due to amplified protein synthesis rates and machinery $[3,4]$. These changes, proceeding as part of the compensatory mechanism, normalize wall stress and optimize contractility without the ability to increase cell number [2].

Other receptors such as $G$ protein-coupled receptors (GPCRs) and receptor tyrosine kinases (RTKs) are known to be activated by autocrine and paracrine factors released during mechanical stress and can function in concert with integrins to activate downstream kinase pathways such as phosphotidylinositol-3-kinase (PI3K). For instance, humoral factors released during hemodynamic overload activate $\beta$-adrenoreceptors and growth factor receptors which may elicit downstream signaling through synergistic integrins. GPCRs activate matrix metalloproteinases (MMPs), MMP-2, MMP-7, MMP-9, and a disintegrin and metalloproteinases (ADAMs), ADAM-12, and ADAM-17. MMPs cleave ECM proteins, resulting in activation of hypertrophic signaling and induction of fetal genes. One recent study showed angiotensin II-induced GPCRs induce MMP-7 which transcriptionally activates ADAM-12. This signaling axis is required for angiotensin-induced hypertrophic signaling [5].

However, mechanical stimulation alone also begets hypertrophic growth [6] through integrin activation [7]. In fact, simply stretching isolated cardiomyocytes results in immediate-early gene and fetal gene induction [8], protooncogene expression [9], and increased protein synthesis [10]. In a feline model of hemodynamic overload, simultaneous inhibition of $\alpha$ - and $\beta$-adrenoreceptors during pressure overload did not affect hypertrophic growth [6]. Therefore, growth can be directly mediated by mechanical stimulation of the myocardium, and integrins are the necessary receptors that physically sense and respond to stretch [11].

\section{Integrin Heterodimers in the Heart}

Integrins are a class of heterodimeric transmembrane proteins made up of the noncovalent interaction of $\alpha$ and $\beta$ subunits. Extracellular domains function for adhesion and ligand recognition, and upon activation, short cytoplasmic domains of $\beta$ subunits, which lack kinase function, physically connect to the cytoskeleton and recruit proteins for signaling [12]. The integrin subunits consist of at least $18 \alpha$ subunits and $8 \beta$ subunits, which in various combinations make up 24 different discovered integrin heterodimers [12]. These heterodimers elicit varying functions and are expressed in a tissue-specific manner [11]. The main integrin heterodimers on the cardiomyocyte surface for activation during hypertrophy are $\alpha_{5} \beta_{1}$ and $\alpha_{v} \beta_{3}$. As physical links between the intracellular cytoskeleton and either the extracellular matrix or the adjacent cardiomyocyte, integrins are regulators of adhesion and mechanotransduction. Integrins are basally expressed in the intercalated disc [13] and costameres of cardiomyocytes $[14,15]$. Intercalated discs are protein complexes at the $\mathrm{Z}$ lines of cardiomyocytes, providing sites of cell-cell interactions. Costameres are adjacent to the $\mathrm{Z}$ lines in cardiomyocytes and offer connection sites for cellextracellular matrix interactions. Most integrin heterodimers bind all ECM proteins while the $\alpha_{5} \beta_{1}$ only binds to fibronectin [16]. The importance of integrins for mechanotransduction and/or adhesion through these interactions is evidenced by integrin upregulation in the intercalated disc upon damage to the myocardium to make new extracellular matrix contacts for intracellular signaling $[17,18]$. Both $\beta_{3}$ and $\beta_{1}$ integrins have been identified at intercalated discs $[17-20]$ and costameres $[15,21]$. To mediate signaling, $\beta_{1}$ integrin translocates to the intercalated disc in damaged areas of the myocardium after infarct [17] while $\beta_{3}$ integrin is upregulated to the cell surface in RGD-stimulated adult cardiomyocytes [22]. Additionally, $\alpha_{5} \beta_{1}$ has been shown to be important for adherence of cardiomyocytes [11] and is necessary for growth in rat neonatal cardiomyocytes [23-25].

Prior to mechanical stimulation, integrins remain freely mobile singular heterodimers on the cell surface, meaning they do not have a constitutive connection to extracellular matrix proteins or cytoskeletal proteins. Integrins that respond to mechanical stretch of the myocardium, namely, $\alpha_{5} \beta_{1}$ and, mediate their signals by recognizing the specific amino acid sequence Arg-Gly-Asp (RGD) in ECM proteins, such as collagen, vitronectin, and fibronectin [16]. Although various integrin subunits have specificities to RGD motifs of various ECM proteins including fibronectin, vitronectin, and collagen, many of them have recognition motifs in the non-RGD motifs of ECM proteins, however the $\alpha_{1}, \alpha_{5}$, and $\beta_{3}$ subunits have RGD specificity [12]. The $\beta_{1}$ subunit is more promiscuous, and its role in binding to RGD is dependent on the associated $\alpha$ subunit [12]. For example, by pairing with $\alpha_{5}$ subunit, $\beta_{1}$ integrin interacts primarily with the RGD motif of fibronectin. In intact myocardium, tortional stress induced in the tissue from increased blood pressure causes structural alterations of ECM proteins which results in exposure of their RGD motifs. These extracellular matrix proteins are also genetically upregulated and secreted with hypertrophic induction in the immediate early gene response of cardiomyocytes [22, 26, 27]. Concurrently, integrin activation at the cardiomyocyte cell surface also increases [19].

Since integrins are positioned to sense stretch of the myocardium, both the $\beta_{1}$ and $\beta_{3}$ integrins have roles in hypertrophic growth. During hypertrophic induction, several integrin subunits are upregulated and/or activated: $\alpha_{1}, \alpha_{5}, \beta_{1}$, and $\beta_{3}[19,28]$, suggesting their role in promoting cardiac hypertrophy. Genetic ablation of either $\beta_{3}$ or $\beta_{1}$ integrins inhibits pressure-induced hypertrophic signaling, resulting in reduced cardiac output with increased mortality and heart failure $[18,20]$. It is clear $\beta_{1}$ integrin plays a role in 
$\beta$-adrenergic receptor-induced hypertrophy [23-25, 29-31]. In neonatal rat ventricular cardiomyocytes, overexpression of $\beta_{1}$ integrin activates expression of the immediate early gene, atrial natriuretic factor (ANF). Conversely, blocking $\beta_{1}$ integrin prevents ANF expression during $\alpha_{1}$-adrenergic stimulation [23]. A heterozygous conditional $\beta_{1}$ knockout mouse subjected to isoproterenol exhibits less hypertrophic growth and more apoptosis (TUNEL-positive nuclei) compared to wild-type mice [29]. Additionally, $\beta_{1}$ integrin is involved in another humoral hypertrophic pathway, as blocking antibodies for $\beta_{1}$ integrin in a stretch model of adult rat cardiomyocytes reduced Ang II secretion [31]. However, expression of a truncated form of $\beta_{1}$ integrin without the cytoplasmic domain in RGD-stimulated adult cardiomyocytes renders $\beta_{1}$ integrin incapable of recruiting specific focal adhesion molecules, though focal adhesion complex (FAC) formation itself is not affected [19]. In the case of $\beta_{3}$ integrin, our recent work demonstrates that FAC formation and subsequent downstream signaling in pressureoverloaded myocardium occurs through this integrin activation [20]. That is, transverse aortic restriction in $\beta_{3}$ integrin knockout mice results in a deficiency of compensatory hypertrophic growth, evidenced by stunted hypertrophic growth, decreased ventricular function and geometry, and increased mortality with compromised survival signaling [20]. Furthermore, restriction of p70 ribosomal S6 kinase activation in cell culture is observed when inhibiting $\beta_{3}$ integrin signaling but not with $\beta_{1}$ integrin inhibition with specific blocking antibodies [32]. This observation indicates distinct effects of these two integrin subtypes.

Upon integrin activation from pressure-induced hypertrophy, multiple downstream signaling cascades mediate protein synthesis, survival, and gene expression: (i) there is evidence both $\beta_{1}$ and $\beta_{3}$ integrins initiate downstream survival signaling utilizing PI3K/Akt and ubiquitination activation [30, 33, 34], (ii) mechanical stimulation of cardiomyocytes and pressure overload in vivo have been shown to initiate FAC formation $[7,22]$ and downstream signaling via Ras-ERK1/2 and Akt [35], (iii) $\beta_{3}$ integrin activates caspase inhibiting molecules for survival signaling in smooth muscle cells [36], cardiomyocytes [20], and hypertrophic myocardium [32, 33], and (iv) it is well documented that hypertrophic induction of integrins results in the activation of mammalian target of rapamycin (mTOR) for increased protein synthesis and cell growth [32]. The survival signaling regulated by ubiquitination downstream of integrin activation is discussed in more detail below.

\section{Integrins Link Mechanical and Biochemical Signals via Activation of Nonreceptor Tyrosine Kinases}

Once activated after recognition of extracellular matrix proteins, integrins cluster on the cell surface, associate with cytoskeletal proteins, and recruit signaling molecules onto the actin cytoskeleton to form a complex of scaffolding proteins and kinases called a FAC. Since the cytoplasmic tails of integrins do not possess kinase function on their own, recruitment of kinase molecules into the FAC is required for initiation of intracellular signaling and downstream gene expression (Reviewed in [37]). In this way, integrins are a physical link between the extracellular matrix and the intracellular cytoskeleton and transduce mechanical signals into intracellular biochemical signals. The FAC is made up of nonreceptor tyrosine kinases (NTKs) [7, 22], such as FAK, Pyk2, cSrc, cSrc-kinase (Csk), c-Cbl and other Ser/Thr kinases, that is, PI3K [38] and PKC [39]. FAK is one of the first kinases recruited to the $\beta$ integrin cytoplasmic tail upon stimulation [40]. After autophosphorylation of Tyr397-FAK, SH2 domain-containing proteins are recruited, which include cSrc [41], Nck [42], and PI3K [43]. With recruitment of protein kinases to this complex, intracellular kinase cascades are then activated for growth and survival signaling and gene expression (Figure 1).

The FAK/Src complex mediates hypertrophic growth and survival signaling in the adult rat heart activated by mechanical stimulation from load/pressure [22, 43]. Evidence shows integrin activation of FAK requires Src while GPCR activation of FAK is Src-independent [44]. FAK phosphorylation and translocation in stretch-induced neonatal rat cardiomyocytes induces immediate-early gene expression and hypertrophic signaling. With $5-20 \%$ cyclic stretch, Tyr397-FAK is increased, and FAK translocates from around the nucleus to the myofilaments to activate downstream signaling, including expression of atrial natriuretic factor (ANF) [44]. Therefore, both recruitment and activation of NTKs are critical for the mediation of hypertrophic signaling.

\section{5. $\beta_{1}$ Integrin Signals through the $\beta$-Adrenergic Receptor}

At intercalated discs and costameres, $\beta_{1}$ integrin engages with several critical proteins (illustrated in Figure 1). The focal adhesion protein kindlin-2 is concentrated at intercalated discs and costameres [45]. Kindlin-2 has been proven an important molecule for integrin-mediated adhesion [46] and intercalated disc functionality [47] and has also been shown to activate the major survival kinase integrin-linked kinase (ILK), which binds the cytoplasmic tail of $\beta_{1}$ integrins to transduce biomechanical signals received by the integrin into biochemical signaling within the cardiomyocyte. Since cardiac-specific knockout of ILK causes spontaneous cardiac failure while cardiac-specific knockouts of $\beta_{1}$ integrin, melusin, or FAK require the stress of pressure overload to decompensate to cardiac failure, ILK likely has other signaling effectors to mediate several critical pathways [48, 49]. No investigation in cardiomyocytes of the activation of ILK by $\beta_{3}$ integrin has been published to date. The activation of Akt by ILK has been shown to be necessary for cardiomyocyte contractility, and phosphorylation at $S 473$ of Akt was abolished in $i l k^{-/-}$hearts. The crucial role of ILK in normal cardiac function could be explained by its activation of Akt and because ILK transduces signals from not only mechanical stimulation but also growth factors and cytokines [49]. 


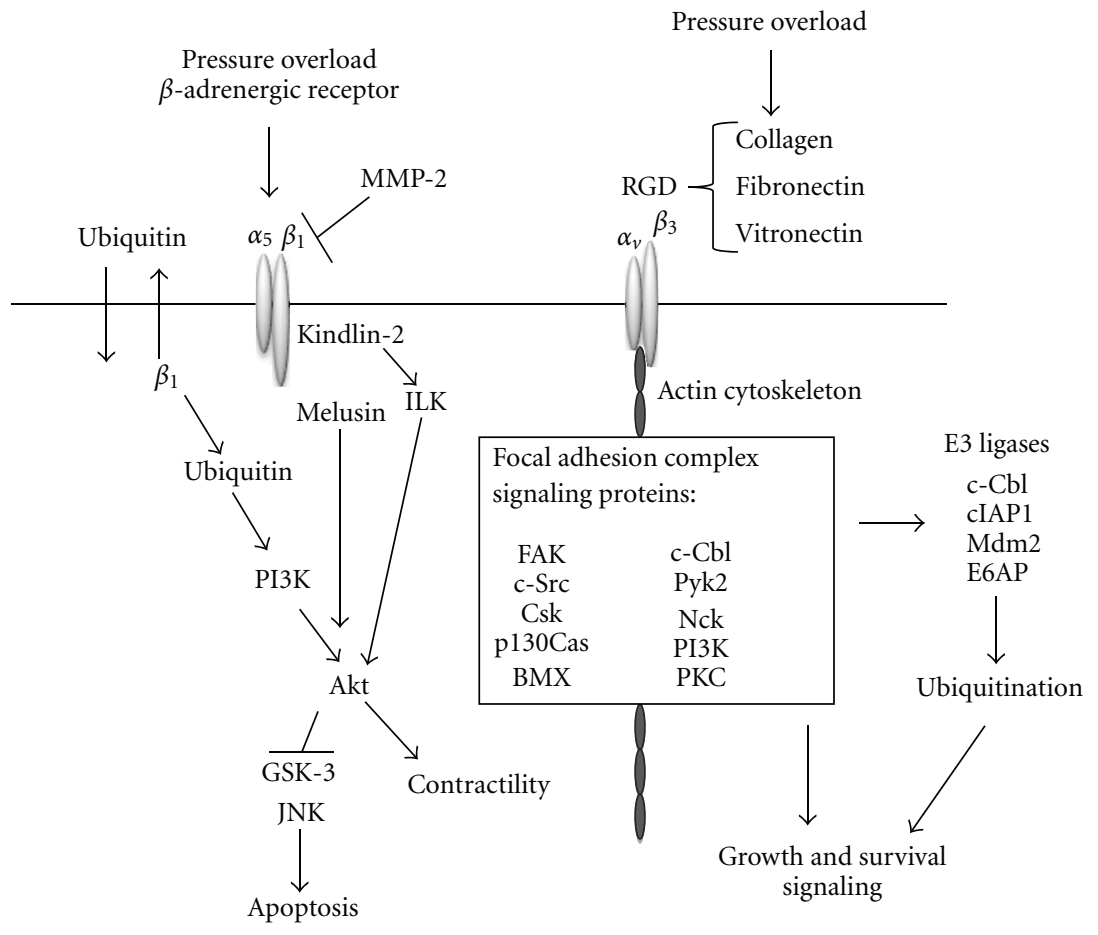

FIGURE 1: Integrin signaling in hypertrophic growth (see text for details).

$\beta_{1}$ integrins also interact with the muscle-specific protein melusin [15]. Genetic disruption of melusin does not affect humoral hypertrophic pathways elicited by angiotensin II or phenylephrine (PE), but melusin is required for pressureinduced hypertrophy [50]. $\beta$-adrenergic receptor stimulation in adult cardiomyocytes results in apoptosis downstream of both GSK3 $\beta$ and c-Jun N-terminal kinase (JNK) activation, which is mediated by the interference of $\beta_{1}$ integrin with the matrix metalloproteinase-2 (MMP-2) [51]; in fact, blocking MMP-2 activation ablates myocardial remodeling and dysfunction during chronic pressure overload [52]. Similarly, disrupting the gene for melusin inhibits Akt-mediated GSK3 $\beta$ phosphorylation and subsequent inhibition during pressure overload [50] while overexpressing melusin inhibits pressure-overload induced apoptosis [53].

\section{Ubiquitin-Mediated Survival Signaling Downstream of $\beta_{1}$ Integrin}

Both $\beta_{1}$ integrin activation [30] and ubiquitin expression [34] can activate the PI3K/Akt pathway to inhibit apoptosis. Ubiquitin was recently found to be excreted by adult cardiomyocytes undergoing $\beta$-adrenergic stimulation by isoproterenol. The ubiquitin is also taken in by the cardiomyocytes and is required for the PI3K-dependent antiapoptotic pathways activated [34]. As described, stimulating $\beta$-adrenergic receptor in adult cardiomyocytes induces apoptosis, measured by terminal deoxynucleotidyl transferasemediated nick end labelling (TUNEL) staining, by activating GSK3 $\beta$ for subsequent JNK activation [49]. Addition of ubiquitin to the media of cardiomyocytes undergoing $\beta$ adrenergic receptor inhibited apoptosis, JNK activation, and cytochrome $\mathrm{c}$ release; further PI3K activation was required [40], since PI3K activates Akt, which directly inhibits GSK3 $\beta$. Though not fully investigated, these data indicate the possible link between $\beta_{1}$ integrin and ubiquitin in antiapoptotic signaling.

\section{Survival Signaling Downstream of $\beta_{3}$ Integrin and Ubiquitination}

In $\beta_{3}$ integrin signaling, hypertrophic stimulation leads to FAC formation, ubiquitination of specific proteins, and $\mathrm{NF} \kappa \mathrm{B}$ activation [20]. Synonymous with integrin-mediated signaling, molecules involved in the ubiquitin-mediated pathway are recruited to the cytoskeleton in order to elicit downstream signaling. $\beta_{3}$ integrin upregulates ubiquitination near intercalated discs of cardiomyocytes within the first week of pressure overload [20]; this is the time period of tyrosine kinase activation downstream of FAC formation $[7,19,22]$. Through specific ubiquitination of the inhibitor of $\kappa \mathrm{B}(\mathrm{I} \kappa \mathrm{B})$ during pressure overload of the heart, $\mathrm{NF} \kappa \mathrm{B}$ is activated and transcribes the cellular inhibitor of apoptosis protein 1 (cIAP1) [20]. Accordingly, $\mathrm{NF} \kappa \mathrm{B}$ is also regulated by FAK activation during mechanical stimulation of cardiomyocytes [54]. NF $\kappa \mathrm{B}$ functions as a survival transcription factor known to be required for hypertrophic growth [55], and one of its transcripts is the cellular inhibitor of apoptosis (cIAP), an E3 ligase that causes ubiquitin-mediated removal of caspases [56]. E3 ligases are the enzymes responsible for recognizing and tagging specific proteins with ubiquitin, and 


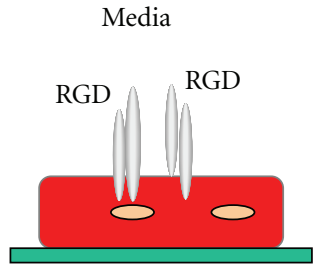

2D cell culture

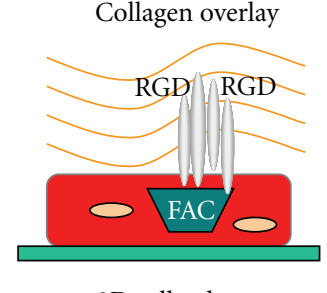

$3 \mathrm{D}$ cell culture
FIGURE 2: Diagram of the collagen overlay model for integrin stimulation in cardiomyocytes with RGD peptide. Cardiomyocytes are plated on laminin-coated plates in media desired for optimal experimental conditions. For integrin stimulation under twodimensional (2D) conditions (left side of the figure), RGD peptide is added directly in the media to stimulate integrin but with no FAC. For integrin stimulation and FAC formation, (figure on the right), media are removed and replaced with the type I collagen matrix mixed with RGD peptide and allowed to polymerize. Integrins are represented by grey cylinders in the figure.

several are upregulated during pressure-induced hypertrophy, including MDM2, cIAP1, c-Cbl, and E6AP [57]. During pressure overload and RGD stimulation in adult cardiomyocytes, $\beta_{3}$ integrin regulates cIAP1 expression. Consequently, the lack of cIAP1 expression correlates with increased caspase 3 activation [20]. A similar antiapoptotic pathway has also been identified in smooth muscle cells [36]. Therefore, cardiomyocytes are able to sense the pressure/stretch stimulus from pressure overload and regulate intracellular signaling through integrin-mediated ubiquitination and elimination of targeted proteins for their survival.

c-Cbl is an E3 ligase for both RTKs and NTKs [58]. Confocal microscopic analysis of c-Cbl localization at $48 \mathrm{~h}$ of pressure overload shows that upregulation of c-Cbl occurs at intercalated discs [57]. In addition to RTKs, c-Cbl has been shown to ubiquitinate other receptors, including GPCRs [59] during hypertrophy. Thus, c-Cbl is linked to the endocytosis and recycling processes of cell surface receptors. Additionally, $\mathrm{c}-\mathrm{Cbl}$ is also a kinase and scaffold protein in the FAC, so localization of $\mathrm{c}-\mathrm{Cbl}$ to the intercalated discs during pressure overload is indicative of its role in both FAC formation and its role in ubiquitination. Ubiquitination itself may also play a role in FAC formation during pressure overload. Significantly, ubiquitination is not enhanced without FAC formation. That is, integrin stimulation with RGD in the two-dimensional cardiomyocyte model, that fails to form FAC was not accompanied by the enhanced ubiquitination whereas the three-dimensional (3D) model (detailed below) of RGD-stimulated integrin activation exhibits both FAC formation and enhanced ubiquitination [20]. These data indicate the importance of integrin activation and FAC formation for the elimination of specific target proteins via ubiquitination as part of the cell survival mechanism.

\section{Integrin-Activating Cell Culture Model}

As mentioned, integrin activation and FAC formation can be recapitulated in vitro by embedding laminin-plated adult cardiomyocytes in a collagen matrix with the integrinstimulating peptide RGD, referred to as a $3 \mathrm{D}$ model (Figure 2). This model specifically activates integrins in a similar manner as in intact tissue when the RGD motif is exposed in the extracellular matrix. When the collagen polymerizes, the embedded RGD peptide can tether to the collagen matrix to induce the integrin heterodimers on the cell surface, the most prominent being $\beta_{3}$ and $\alpha_{5} \beta_{1}$ [22]. To decipher signaling that is independent of the FAC formation, a 2D model can be used where RGD is added directly to media of laminin-plated cardiomyocytes. Without the stabilization provided by the polymerized collagen, the RGD is absorbed by the cardiomyocytes [32]. Devoid of the semisolid collagen matrix, RGD simply engages integrins without inducing FAC formation in the $2 \mathrm{D}$ model because integrins are unable to cluster and recruit focal adhesion proteins onto the cytoskeletal complex [22,32]. Therefore, many integrin-mediated pathways requiring FAC formation for their activation are silent in this 2D model [22], such as ubiquitination [20]. On the other hand, the 3D collagen model is able to recapitulate the recruitment and activation of the $\beta_{3}$ integrin and focal adhesion proteins, such as Src, FAK, Nck, Shc, and p130Cas, as observed in the hypertrophic animal models [22]. Importantly, introducing RGD to laminincoated cardiomyocytes without collagen (2D model) does not cause the recruitment of these proteins to the FAC. It is also apparent that FAK is not phosphorylated during RGD treatment without FAC formation [22], indicating activation of FAK requires its recruitment to FAC. These studies lend to both pharmacological and adenoviral manipulations of cardiomyocytes, and methods have also been optimized to utilize cardiomyocytes from knockout murine models [20]. These cell culture models are important tools to decipher the intracellular signaling of integrins in cardiomyocytes.

\section{Conclusions}

Both $\beta_{1}$ and $\beta_{3}$ integrins are required for compensatory hypertrophic growth in response to pressure overload of the heart. Integrins interact with a complex network of proteins, which new studies continue to show are necessary for normal or hypertrophic cardiac function. The complex network of proteins transducing $\beta_{1}$ integrin-mediated survival and growth signaling continues to grow. This includes a newly discovered interaction with ubiquitin, shown to activate PI3K/Akt signaling downstream of $\beta_{1}$ integrin activation. Moreover, the importance of $\beta_{3}$ integrins in ubiquitin-mediated removal of proapoptotic proteins for the survival of hypertrophying cardiomyocytes is a recent finding, and additional studies will likely decipher how this integrin subtype is tied into ubiquitination in the heart. Since integrins, PI3K/Akt, and ubiquitin signaling are each required for hypertrophic induction, continuing to study their interdependence will lead to better understanding of cardiac function during hemodynamic overload. Further study of integrin signaling may help decipher the various roles integrins play in cardiac performance and aid in the identification of potential drug targets against cardiac failure. 


\section{Acknowledgments}

This work was supported by the National Institutes of Health [RHL092124A to D. Kuppuswamy], by AHA predoctoral fellowship 0615468U (to R.K. Harton), and by NIH predoctoral fellowship NIH T32HL07260 (to R.K.H.). The authors thank Dr. John McKillop for careful reading of the paper.

\section{References}

[1] N. Frey and E. N. Olson, "Cardiac hypertrophy: the good, the bad, and the ugly," Annual Review of Physiology, vol. 65, pp. 45-79, 2003.

[2] C. Ruwhof and A. van der Laarse, "Mechanical stress-induced cardiac hypertrophy: mechanisms and signal transduction pathways," Cardiovascular Research, vol. 47, no. 1, pp. 23-37, 2000.

[3] W. J. Tuxworth, H. Shiraishi, P. C. Moschella, K. Yamane, P. J. McDermott, and D. Kuppuswamy, "Translational activation of 5'-TOP mRNA in pressure overload myocardium," Basic Research in Cardiology, vol. 103, no. 1, pp. 41-53, 2008.

[4] H. E. Morgan and C. J. Beinlich, "Contributions of increased efficiency and capacity of protein synthesis to rapid cardiac growth," Molecular and Cellular Biochemistry, vol. 176, no. 1-2, pp. 145-151, 1997.

[5] X. Wang, F. L. Chow, T. Oka et al., "Matrix metalloproteinase7 and ADAM-12 (a disintegrin and metalloproteinase-12) define a signaling axis in agonist-induced hypertension and cardiac hypertrophy," Circulation, vol. 119, no. 18, pp. 24802489, 2009.

[6] G. Cooper, R. L. Kent, C. E. Uboh, E. W. Thompson, and T. A. Marino, "Hemodynamic versus adrenergic control of cat right ventricular hypertrophy," Journal of Clinical Investigation, vol. 75, no. 5, pp. 1403-1414, 1985.

[7] D. Kuppuswamy, C. Kerr, T. Narishige, V. S. Kasi, D. R. Menick, and G. Cooper, "Association of tyrosine-phosphorylated cSrc with the cytoskeleton of hypertrophying myocardium," Journal of Biological Chemistry, vol. 272, no. 7, pp. 4500-4508, 1997.

[8] J. I. Sadoshima, L. Jahn, T. Takahashi, T. J. Kulik, and S. Izumo, "Molecular characterization of the stretch-induced adaptation of cultured cardiac cells. An in vitro model of load-induced cardiac hypertrophy," Journal of Biological Chemistry, vol. 267, no. 15, pp. 10551-10560, 1992.

[9] I. Komuro, T. Kaida, Y. Shibazaki et al., "Stretching cardiac myocytes stimulates protooncogene expression," Journal of Biological Chemistry, vol. 265, no. 7, pp. 3595-3598, 1990.

[10] Y. Kira, T. Nakaoka, E. Hashimoto, F. Okabe, S. Asano, and I. Sekine, "Effect of long-term cyclic mechanical load on protein synthesis and morphological changes in cultured myocardial cells from neonatal rat," Cardiovascular Drugs and Therapy, vol. 8, no. 2, pp. 251-262, 1994.

[11] R. S. Ross and T. K. Borg, "Integrins and the myocardium," Circulation Research, vol. 88, no. 11, pp. 1112-1119, 2001.

[12] R. O. Hynes, "Integrins: bidirectional, allosteric signaling machines," Cell, vol. 110, no. 6, pp. 673-687, 2002.

[13] J. Q. Zhang, B. Elzey, G. Williams, S. Lu, D. J. Law, and R. Horowits, "Ultrastructural and biochemical localization of NRAP at the interface between myofibrils and intercalated disks in the mouse heart," Biochemistry, vol. 40, no. 49, pp. 14898 14906, 2001.
[14] T. K. Borg, E. C. Goldsmith, R. Price, W. Carver, L. Terracio, and A. M. Samarel, "Specialization at the $\mathrm{Z}$ line of cardiac myocytes," Cardiovascular Research, vol. 46, no. 2, pp. 277$285,2000$.

[15] M. Brancaccio, S. Guazzone, N. Menini et al., "Melusin is a new muscle-specific interactor for $\beta_{1}$ integrin cytoplasmic domain," Journal of Biological Chemistry, vol. 274, no. 41, pp. 29282-29288, 1999.

[16] B. Wehrle-Haller and B. A. Imhof, "Integrin-dependent pathologies," Journal of Pathology, vol. 200, no. 4, pp. 481-487, 2003.

[17] T. Matsushita, M. Oyamada, K. Fujimoto et al., "Remodeling of cell-cell and cell-extracellular matrix interactions at the border zone of rat myocardial infarcts," Circulation Research, vol. 85, no. 11, pp. 1046-1055, 1999.

[18] C. J. Babbitt, S. Y. Shai, A. E. Harpf, C. G. Pham, and R. S. Ross, "Modulation of integrins and integrin signaling molecules in the pressure-loaded murine ventricle," Histochemistry and Cell Biology, vol. 118, no. 6, pp. 431-439, 2002.

[19] C. D. Willey, S. Balasubramanian, M. C. Rodríguez Rosas, R. S. Ross, and D. Kuppuswamy, "Focal complex formation in adult cardiomyocytes is accompanied by the activation of $\beta 3$ integrin and c-Src," Journal of Molecular and Cellular Cardiology, vol. 35, no. 6, pp. 671-683, 2003.

[20] R. K. Johnston, S. Balasubramanian, H. Kasiganesan, C. F. Baicu, M. R. Zile, and D. Kuppuswamy, " $\beta_{3}$ integrin-mediated ubiquitination activates survival signaling during myocardial hypertrophy," FASEB Journal, vol. 23, no. 8, pp. 2759-2771, 2009.

[21] T. Nagai, M. Laser, C. F. Baicu, M. R. Zile, G. Cooper, and D. Kuppuswamy, " $\beta_{3}$-integrin-mediated focal adhesion complex formation: adult cardiocytes embedded in three-dimensional polymer matrices," American Journal of Cardiology, vol. 83, no. 12A, pp. 38H-43H, 1999.

[22] M. Laser, C. D. Willey, W. Jiang et al., "Integrin activation and focal complex formation in cardiac hypertrophy," Journal of Biological Chemistry, vol. 275, no. 45, pp. 35624-35630, 2000.

[23] R. S. Ross, C. Pham, S. Y. Shai et al., " $\beta_{1}$ Integrins participate in the hypertrophic response of rat ventricular myocytes," Circulation Research, vol. 82, no. 11, pp. 1160-1172, 1998.

[24] E. Ogawa, Y. Saito, M. Harada et al., "Outside-in signalling of fibronectin stimulates cardiomyocyte hypertrophy in cultured neonatal rat ventricular myocytes," Journal of Molecular and Cellular Cardiology, vol. 32, no. 5, pp. 765-776, 2000.

[25] L. K. Hornberger, S. Singhroy, T. Cavalle-Garrido, W. Tsang, F. Keeley, and M. Rabinovitch, "Synthesis of extracellular matrix and adhesion through $\beta_{1}$ integrins are critical for fetal ventricular myocyte proliferation," Circulation Research, vol. 87, no. 6, pp. 508-515, 2000.

[26] S. P. Blatti, D. N. Foster, G. Ranganathan, H. L. Moses, and M. J. Getz, "Induction of fibronectin gene transcription and mRNA is a primary response to growth-factor stimulation of AKR-2B cells," Proceedings of the National Academy of Sciences of the United States of America, vol. 85, no. 4, pp. 1119-1123, 1988.

[27] R. P. Ryseck, H. MacDonald-Bravo, M. Zerial, and R. Bravo, "Coordinate induction of fibronectin, fibronectin receptor, tropomyosin, and actin genes in serum-stimulated fibroblasts," Experimental Cell Research, vol. 180, no. 2, pp. 537-545, 1989.

[28] L. Terracio, K. Rubin, D. Gullberg et al., "Expression of collagen binding integrins during cardiac development and hypertrophy," Circulation Research, vol. 68, no. 3, pp. 734-744, 1991. 
[29] P. Krishnamurthy, V. Subramanian, M. Singh, and K. Singh, " $\beta 1$ integrins modulate $\beta$-adrenergic receptor-stimulated cardiac myocyte apoptosis and myocardial remodeling," Hypertension, vol. 49, no. 4, pp. 865-872, 2007.

[30] B. Menon, J. N. Johnson, R. S. Ross, M. Singh, and K. Singh, "Glycogen synthase kinase- $3 \beta$ plays a pro-apoptotic role in $\beta$-adrenergic receptor-stimulated apoptosis in adult rat ventricular myocytes: role of $\beta 1$ integrins," Journal of Molecular and Cellular Cardiology, vol. 42, no. 3, pp. 653-661, 2007.

[31] X. Yutao, W. Geru, B. Xiaojun, G. Tao, and M. Aiqun, "Mechanical stretch-induced hypertrophy of neonatal rat ventricular myocytes is mediated by $\beta_{1}$-integrin-microtubule signaling pathways," European Journal of Heart Failure, vol. 8, no. 1, pp. 16-22, 2006.

[32] S. Balasubramanian and D. Kuppuswamy, "RGD-containing peptides activate S6K1 through $\beta_{3}$ integrin in adult cardiac muscle cells," Journal of Biological Chemistry, vol. 278, no. 43, pp. 42214-42224, 2003.

[33] R. K. Johnston, S. Balasubramanian, H. Kasiganesan, C. F. Baicu, M. R. Zile, and D. Kuppuswamy, " $\beta_{3}$ integrin-mediated ubiquitination activates survival signaling during myocardial hypertrophy," FASEB Journal, vol. 23, no. 8, pp. 2759-2771, 2009.

[34] M. Singh, M. Roginskaya, S. Dalal et al., "Extracellular ubiquitin inhibits $\beta$-AR-stimulated apoptosis in cardiac myocytes: role of GSK-3 $\beta$ and mitochondrial pathways," Cardiovascular Research, vol. 86, no. 1, pp. 20-28, 2010.

[35] Y. Iijima, M. Laser, H. Shiraishi et al., "c-Raf/MEK/ERK pathway controls protein kinase C-mediated p70S6K activation in adult cardiac muscle cells," Journal of Biological Chemistry, vol. 277, no. 25, pp. 23065-23075, 2002.

[36] K. von Wnuck Lipinski, P. Keul, N. Ferri et al., "Integrinmediated transcriptional activation of inhibitor of apoptosis proteins protects smooth muscle cells against apoptosis induced by degraded collagen," Circulation Research, vol. 98, no. 12, pp. 1490-1497, 2006.

[37] S. K. Sastry and K. Burridge, "Focal adhesions: a nexus for intracellular signaling and cytoskeletal dynamics," Experimental Cell Research, vol. 261, no. 1, pp. 25-36, 2000.

[38] H. C. Chen, P. A. Appeddu, H. Isoda, and J. L. Guan, "Phosphorylation of tyrosine 397 in focal adhesion kinase is required for binding phosphatidylinositol 3-kinase," Journal of Biological Chemistry, vol. 271, no. 42, pp. 26329-26334, 1996.

[39] M. C. Heidkamp, A. L. Bayer, B. T. Scully, D. M. Eble, and A. M. Samarel, "Activation of focal adhesion kinase by protein kinase $\mathrm{C} \varepsilon$ in neonatal rat ventricular myocytes," American Journal of Physiology, vol. 285, no. 4, pp. H1684-H1696, 2003.

[40] J. L. Guan, J. E. Trevithick, and R. O. Hynes, "Fibronectin/integrin interaction induces tyrosine phosphorylation of a $120-\mathrm{kDa}$ protein," Cell Regulation, vol. 2, no. 11, pp. 951-964, 1991.

[41] M. D. Schaller, J. D. Hildebrand, J. D. Shannon, J. W. Fox, R. R. Vines, and J. T. Parsons, "Autophosphorylation of the focal adhesion kinase, pp $125^{\mathrm{FAK}}$, directs $\mathrm{SH} 2$-dependent binding of pp60 ${ }^{\text {sc }}$," Molecular and Cellular Biology, vol. 14, no. 3, pp. 1680-1688, 1994.

[42] G. G. Choudhury, F. Marra, and H. E. Abboud, "Thrombin stimulates association of src homology domain containing adaptor protein Nek with pp125," American Journal of Physiology, vol. 270, no. 2, pp. F295-F300, 1996.
[43] K. G. Franchini, A. S. Torsoni, P. H. A. Soares, and M. J. A. Saad, "Early activation of the multicomponent signaling complex associated with focal adhesion kinase induced by pressure overload in the rat heart," Circulation Research, vol. 87, no. 7, pp. 558-565, 2000.

[44] A. S. Torsoni, S. S. Constancio, W. Nadruz, S. K. Hanks, and K. G. Franchini, "Focal adhesion kinase is activated and mediates the early hypertrophic response to stretch in cardiac myocytes," Circulation Research, vol. 93, no. 2, pp. 140-147, 2003.

[45] S. Ussar, H. V. Wang, S. Linder, R. Fässler, and M. Moser, "The Kindlins: subcellular localization and expression during murine development," Experimental Cell Research, vol. 312, no. 16, pp. 3142-3151, 2006.

[46] Y. Tu, S. Wu, X. Shi, KA. Chen, and C. Wu, "Migfilin and Mig2 link focal adhesions to filamin and the actin cytoskeleton and function in cell shape modulation," Cell, vol. 113, no. 1, pp. 37-47, 2003.

[47] J. J. Dowling, E. Gibbs, M. Russell et al., "Kindlin-2 is an essential component of intercalated discs and is required for vertebrate cardiac structure and function," Circulation Research, vol. 102, no. 4, pp. 423-431, 2008.

[48] C. J. Hatcher and C. T. Basson, "Disrupted intercalated discs: is kindlin-2 required?" Circulation Research, vol. 102, no. 4, pp. 392-394, 2008.

[49] G. E. Hannigan, J. G. Coles, and S. Dedhar, "Integrin-linked kinase at the heart of cardiac contractility, repair, and disease," Circulation Research, vol. 100, no. 10, pp. 1408-1414, 2007.

[50] M. Brancaccio, L. Fratta, A. Notte et al., "Melusin, a musclespecific integrin $\beta$-interacting protein, is required to prevent cardiac failure in response to chronic pressure overload," Nature Medicine, vol. 9, no. 1, pp. 68-75, 2003.

[51] B. Menon, M. Singh, R. S. Ross, J. N. Johnson, and K. Singh, " $\beta$-adrenergic receptor-stimulated apoptosis in adult cardiac myocytes involves MMP-2-mediated disruption of $\beta$ integrin signaling and mitochondrial pathway," American Journal of Physiology, vol. 290, no. 1, pp. C254-C261, 2006.

[52] J. T. Peterson, H. Hallak, L. Johnson et al., "Matrix metalloproteinase inhibition attenuates left ventricular remodeling and dysfunction in a rat model of progressive heart failure," Circulation, vol. 103, no. 18, pp. 2303-2309, 2001.

[53] M. De Acetis, A. Notte, F. Accornero et al., "Cardiac overexpression of melusin protects from dilated cardiomyopathy due to long-standing pressure overload," Circulation Research, vol. 96, no. 10, pp. 1087-1094, 2005.

[54] D. P. Crosara-Alberto, R. Y. Inoue, and C. R. C. Costa, "FAK signalling mediates NF- $\kappa \mathrm{B}$ activation by mechanical stress in cardiac myocytes," Clinica Chimica Acta, vol. 403, no. 1-2, pp. 81-86, 2009.

[55] Y. Li, T. Ha, X. Gao et al., "NF- $\kappa$ activation is required for the development of cardiac hypertrophy in vivo," American Journal of Physiology, vol. 287, no. 4, pp. H1712-H1720, 2004.

[56] Y. E. Choi, M. Butterworth, S. Malladi, C. S. Duckett, G. M. Cohen, and S. B. Bratton, "The E3 ubiquitin ligase cIAP1 binds and ubiquitinates caspase- 3 and -7 via unique mechanisms at distinct steps in their processing," Journal of Biological Chemistry, vol. 284, no. 19, pp. 12772-12782, 2009.

[57] S. Balasubramanian, S. Mani, H. Shiraishi et al., "Enhanced ubiquitination of cytoskeletal proteins in pressure overloaded myocardium is accompanied by changes in specific E3 ligases," Journal of Molecular and Cellular Cardiology, vol. 41, no. 4, pp. 669-679, 2006. 
[58] C. A. P. Joazeiro, S. S. Wing, H. K. Huang, J. D. Leverson, T. Hunter, and Y. C. Liu, "The tyrosine kinase negative regulator $\mathrm{c}-\mathrm{Cbl}$ as a RING-type, E2-dependent ubiquitinprotein ligase," Science, vol. 286, no. 5438, pp. 309-312, 1999.

[59] R. J. Lefkowitz, "G protein-coupled receptors: III. New roles for receptor kinases and $\beta$-arrestins in receptor signaling and desensitization," Journal of Biological Chemistry, vol. 273, no. 30, pp. 18677-18680, 1998. 

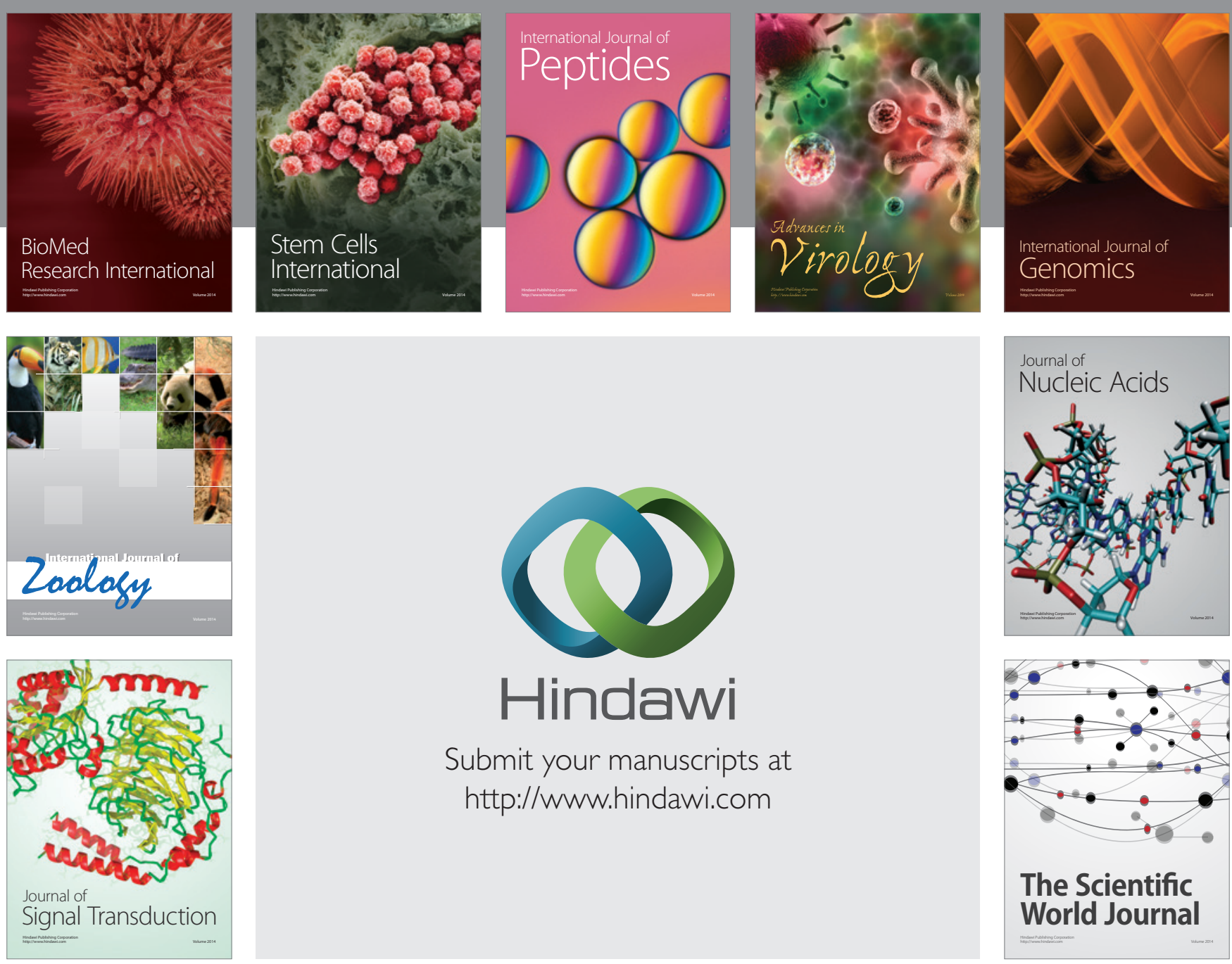

Submit your manuscripts at

http://www.hindawi.com
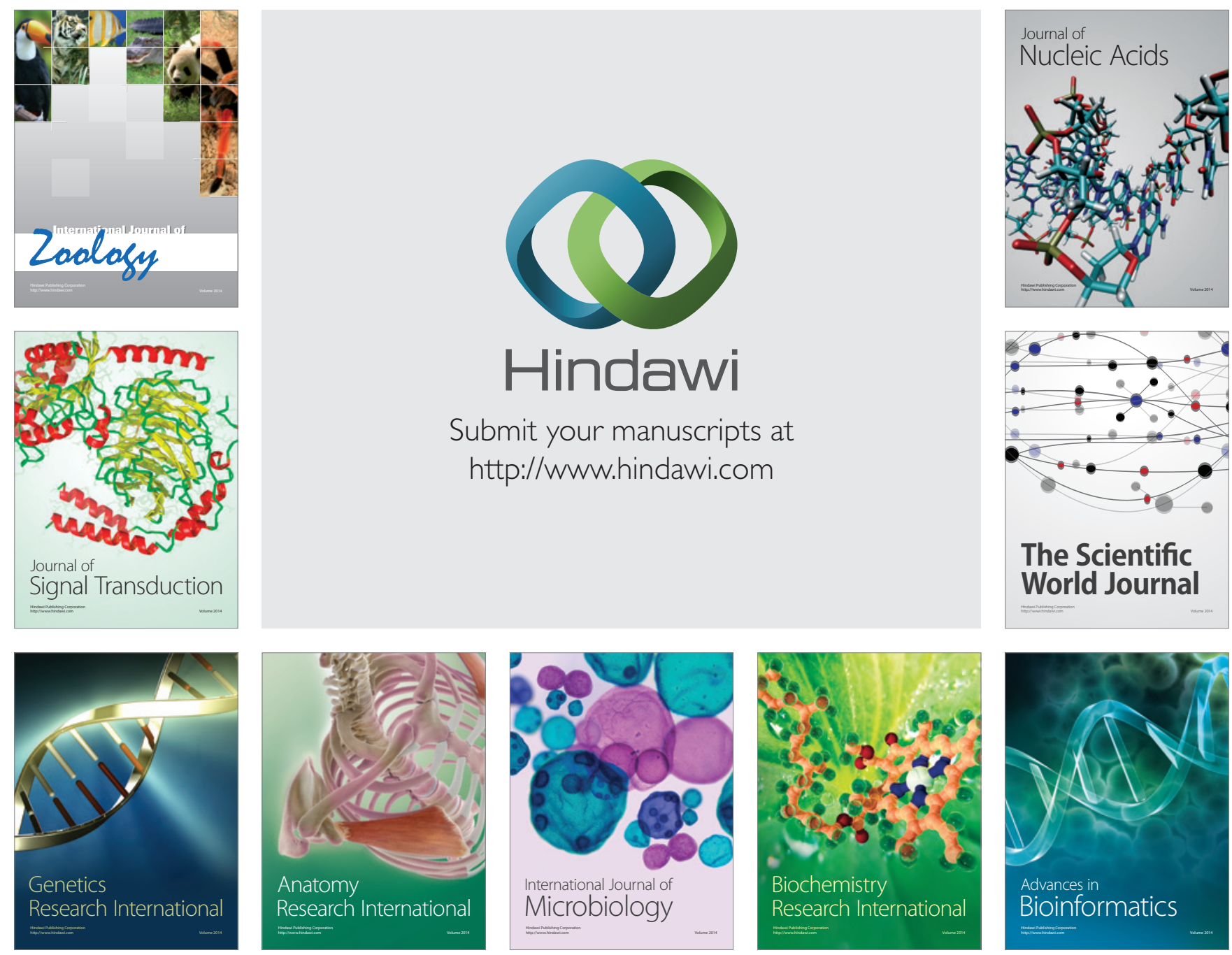

The Scientific World Journal
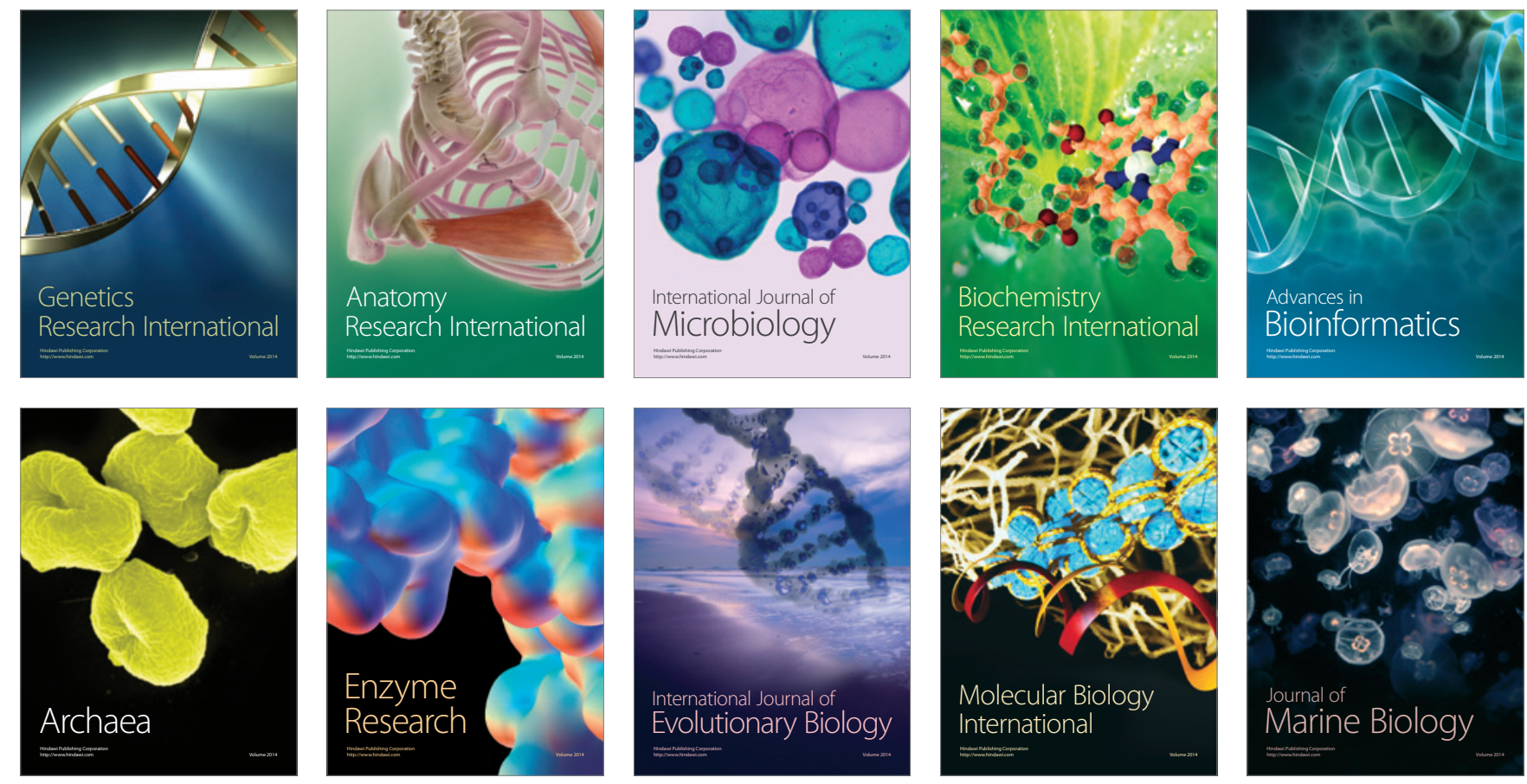